Supplementary information for the article

\title{
Single Molecule Nano-Spectroscopy Visualizes Proton-Transfer Processes within a Zeolite Crystal
}

Zoran Ristanović, Alexey V. Kubarev, Johan Hofkens, Maarten B. J. Roeffaers, ${ }^{*}$ and Bert M. Weckhuysen*

Supplementary Movie 1: The photo-activity of a parent zeolite ZSM-5 crystal in 4-methoxystyrene recorded at $Z=0 \pm 0.3 \mu \mathrm{m}$ surface), $100 \mathrm{~ms}$ per frame. The field of view is $25 \times 25 \mu \mathrm{m}^{2}$.

Supplementary Movie 2: The reactivity of a steamed zeolite ZSM-5 crystal in $30 \mu \mathrm{M}$ solution of 4methoxystyrene in $\mathrm{n}$-heptane, recorded at $\mathrm{Z}=2 \pm 0.3 \mu \mathrm{m}, 30 \mathrm{~ms}$ per frame. The field of view is $25 \times$ $25 \mu \mathrm{m}^{2}$.

\section{Supplementary Figures S1-S8.}


UV-Vis micro-spectroscopy: UV-Vis absorption spectra were measured with an Olympus BX41 upright microscope working in reflectance mode, equipped with a $50 \times 0.5 \mathrm{NA}$ high working-distance microscope objective lens. A $75 \mathrm{~W}$ tungsten lamp was used for illumination. Reflected light was directed to a CCD video camera (ColorView IIIu, Soft Imaging System GmbH) via a 50/50 double viewport tube, and to a UV-Vis spectrometer (AvaSpec-2048TEC, Avantes BV) via a $200 \mu \mathrm{m}$ core fiber.
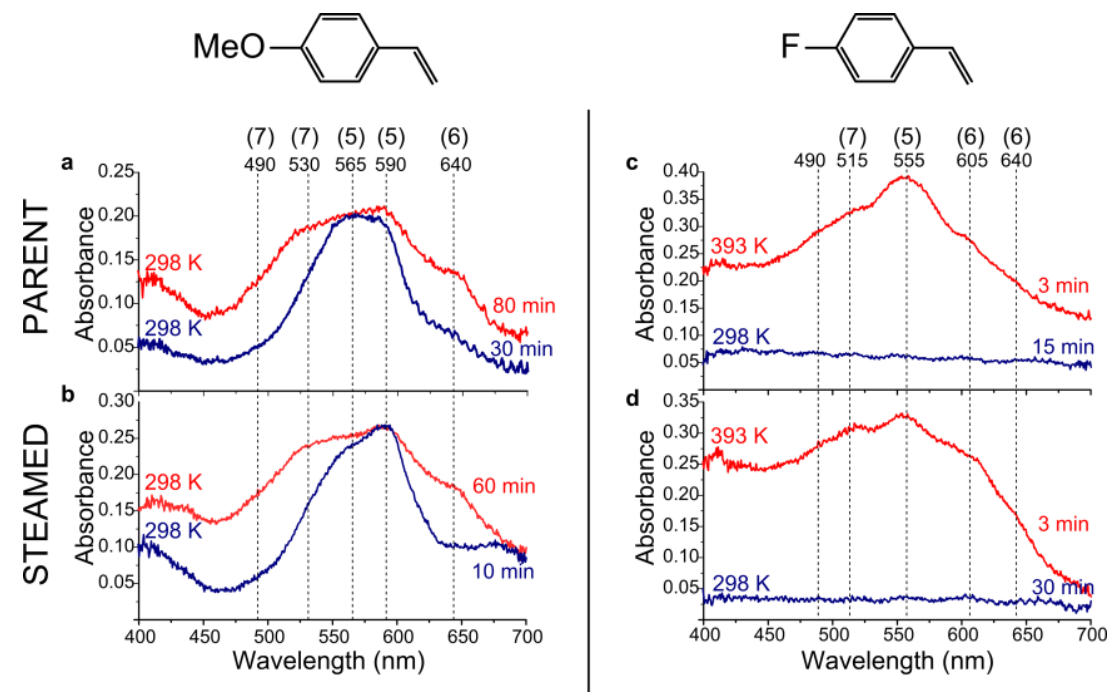

Figure S1. UV-Vis absorption spectra of the studied oligomerization reactions under solvent-free conditions. The spectra were measured with an UV-Vis microscope at the center of zeolite crystals; top panels - parent H-ZSM-5, bottom panels - steamed H-ZSM-5 crystals. a,b) UV-Vis absorption spectra of 4-methoxystyrene oligomers; (a) was recorded for two different crystals. c, d) UV-Vis absorption spectra of 4-fluorostyrene oligomers at room temperature and $393 \mathrm{~K}$. The numbers above the absorption maxima indicate the tentative assignment to the carbocationic species shown in Scheme 1 of the main text of the article.

A notable visual difference in reactivity between the 4-methoxy- and 4-fluorostyrenes is noticed with a UV-Vis microscope. The Brønsted acid-catalyzed oligomerization of 4-methoxystyrene readily proceeds at room temperature, coloring the individual zeolite H-ZSM-5 crystals due to the formation of the stable carbocationic species (Figure S1a,b). ${ }^{1}$ In contrast to 4-methoxystyrene, 4fluorostyrene does not display any significant reactivity at room temperature (Figure S1c,d) due to the high electronegativity of the fluoro-substituent that hinders the proton transfer from the Brønsted acid sites of the zeolite material to the double bond of the styrene moiety. ${ }^{2}$ The difference in reactivity of the tested molecules is visible in the comparison of UV-Vis spectra taken from the reaction products, as summarized in Figure S1. Two distinct absorption bands initially appear for both types of zeolite $\mathrm{H}$ ZSM-5 crystals at $\sim 565 \mathrm{~nm}$ and $590 \mathrm{~nm}$. A similar absorption profile was reported previously; the absorption $\sim 590 \mathrm{~nm}$ was attributed to the linear dimeric carbocation (5) in Scheme 1 of the main text of the article. ${ }^{1,3,4} \mathrm{We}$ attribute these absorption bands to the existence of two isomers of the allylic dimeric carbocation (5). The assignment of the band at $\sim 530 \mathrm{~nm}$ has never been attributed before for 4-methoxystyrene as it appears as a satellite band to the observed linear dimeric species. Sprung and Weckhuysen have recently reported $20-40 \mathrm{~nm}$ shifts in the absorption bands of the cyclic carbocations in the sinusoidal pores of zeolite H-ZSM-5. ${ }^{5}$ Additionally, as the formation of the absorption band follows similar trends as for the trimeric (6) and cyclic species (7), suggesting their bulkier nature, we tentatively attribute this band to the cyclic dimeric species. The absorption originating from the trimeric species (6) was previously proposed, ${ }^{1,5}$ but to date their precise molecular structure has not been identified in the experiments. 
Confocal fluorescence microscopy: The fluorescence microscopy measurements were carried out using an upright Nikon Eclipse 90i confocal laser scanning microscope, equipped with a $100 \times 0.73$ NA dry objective lens. Confocal fluorescence microscopy images were recorded using the excitation from two laser light sources (i.e., $488 \mathrm{~nm}$ and $561 \mathrm{~nm}$ ) connected to a Nikon-Eclipse A1R scanning head equipped with corresponding dichroic mirrors to separate the excitation and emission light. The long axes of the zeolite crystals were aligned perpendicular to the polarization vector of the laser light in order to efficiently excite the fluorescent molecules, as explained in reference. ${ }^{5}$ The light emission was detected in the range of 495-700 $\mathrm{nm}$ by using a spectral detection unit equipped with a diffraction grating and a 32 photomultiplier tube array. The fluorescence spectra obtained by $488 \mathrm{~nm}$ (from 490 $600 \mathrm{~nm}$ ) and $561 \mathrm{~nm}$ lasers (from 570-700 nm) were combined.

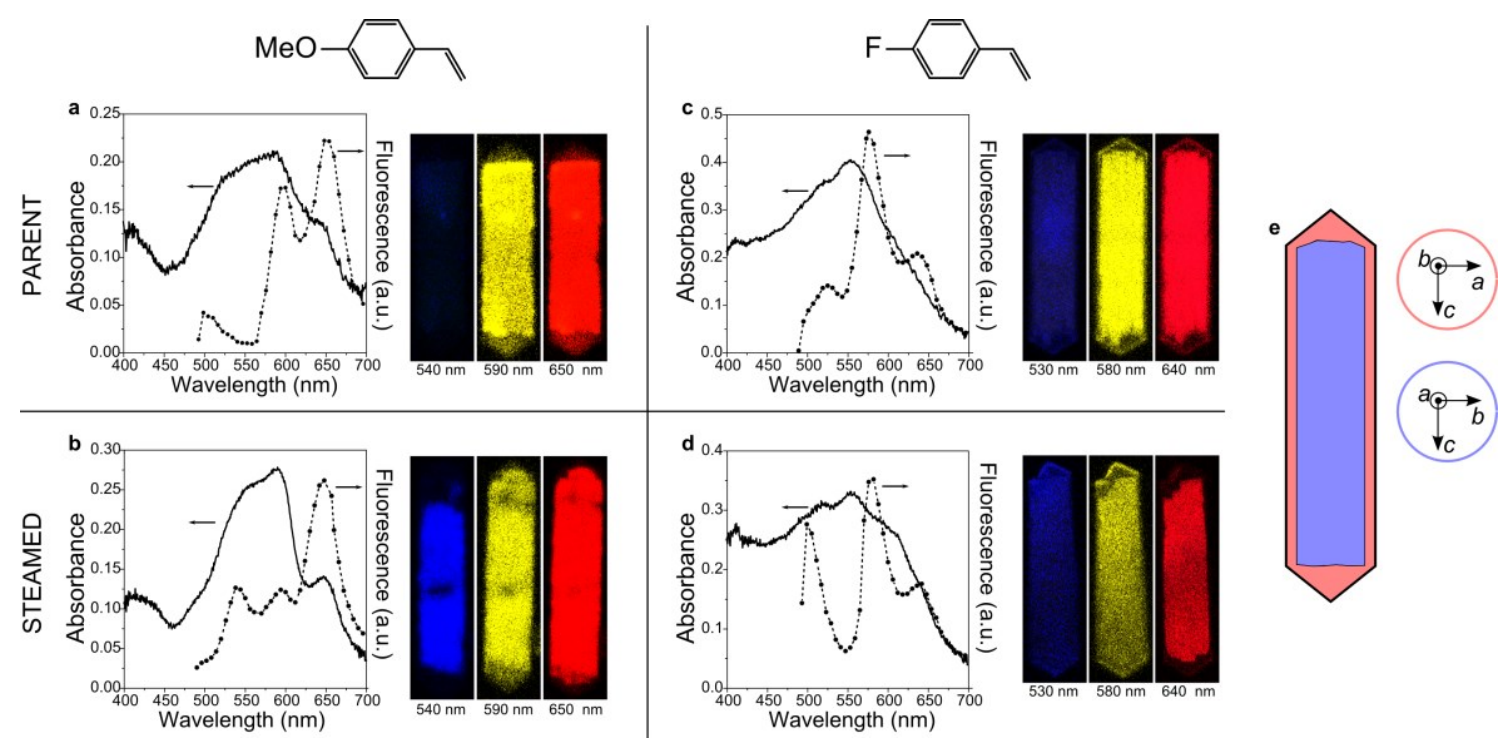

Figure S2. A comparison of the UV-Vis absorption (solid line) and fluorescence spectra (dashed line) of the oligomerization reaction products recorded for the parent (top) and steamed zeolite H-ZSM-5 crystals (bottom). a, b) solvent-free oligomerization of 4-methoxystyrene recorded at room temperature; c, d) solvent-free oligomerization of 4-fluorostyrene recorded at $393 \mathrm{~K}$. Confocal fluorescence microscopy images of the different fluorescent species blue (7), green (5) and red (6) by measuring the fluorescence intensity in a band of $\pm 25 \mathrm{~nm}$ around their maximum emission wavelength. e) Lateral projection of the intergrowth model of an H-ZSM-5 crystal showing $90^{\circ}$ intergrown subunits with different pore orientation; red domain - sinusoidal pores run parallel to the image plane; blue domain - straight pores run parallel to the image plane.

The UV-Vis micro-spectroscopy data with confocal fluorescence microscopy results recorded for both probe reactions as well as for the parent and steamed zeolite H-ZSM-5 crystals are compared in Figure S2. Both emission bands overlap spatially for the top subunits of both the parent and steamed zeolite H-ZSM-5 crystals (Figure S2a,b). At the edge, the $600 \mathrm{~nm}$ band seems to be higher in intensity, as reported in the literature. ${ }^{1}$ The recorded fluorescence predominantly originates from the molecules aligned in the direction of the straight pores directed along $b$ lattice vector (Figure S2e). This was verified in a polarization-sensitive experiment by rotating the zeolite H-ZSM-5 crystal for $90^{\circ} .{ }^{5}$ The emission band at $540 \mathrm{~nm}$ was not present for the majority of the crystals. Thus, based solely on confocal fluorescence microscopy measurements, we have concluded that the concentration of the bulkier trimeric and cyclic dimeric species depends on the inter-particle differences in chemical reactivity, with the formation of the trimeric species being more visible in fluorescence. 
Our experiments suggest that a more intense coloration from increased visible light absorption, of zeolite H-ZSM-5 crystals exposed to 4-methoxystyrene does not directly translate into higher fluorescence intensity. The absence of such dependency clearly indicates the presence of additional non-radiative relaxation pathways. This behavior was most pronounced for 4methoxystyrene and most probably relates to its high reactivity that leads to a locally high concentration of absorbing reaction products. The reported emission bands should be rather used as a guideline for the possible fluorescence species, as the oligomerization kinetics will largely depend on the reaction conditions in different concentration regimes

UV-Vis spectra of H-ZSM-5 crystals: interparticle differences
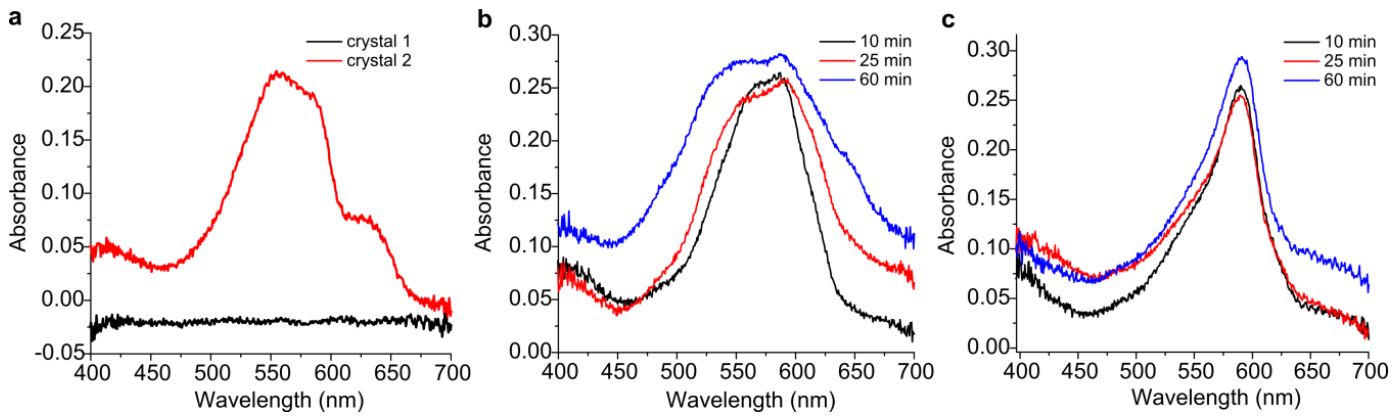

Figure S3. a) UV-Vis spectra of the reactive and non-reactive parent zeolite H-ZSM-5 crystals recorder after $1 \mathrm{~h}$ of a reaction with 4-methoxystyrene. b,c) A time evolution of UV-Vis spectra for two steamed zeolite H-ZSM-5 crystals indicating a difference in the distribution of the colored reaction products.

It is noteworthy that the extent of crystal coloration, time-dependent changes, as well as the number of absorption bands differed significantly from one zeolite H-ZSM-5 crystal to another (Figure S3). The optical microscopy observations indicated large interparticle differences in reactivity that can be most probably attributed to the large surface diffusion resistance and inhomogeneous uptake of reactants. As a consequence, some parent zeolite H-ZSM-5 crystals reached dark-purple coloration, whereas other did not show visible absorption, even after $1 \mathrm{~h}$ of reaction in 4methoxystyrene. 


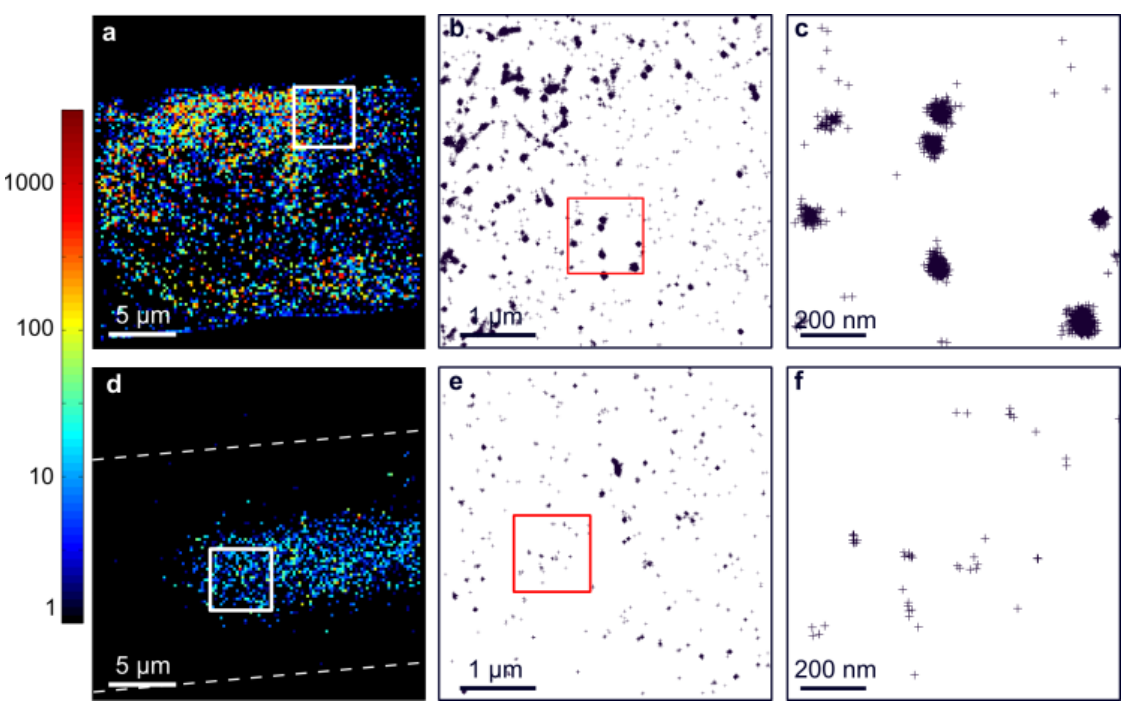

Figure S4. High-resolution single molecule fluorescence microscopy imaging of fluorescent reaction products recorded after $1 \mathrm{~h}$ during $500 \mathrm{~s}$ of the oligomerization of 4-methoxystyrene on a parent zeolite H-ZSM-5 crystal. a) Accumulated map of the number of new fluorescent oligomers formed in a certain region of the zeolite crystal (color bar: number of events per $200 \times 200 \mathrm{~nm}^{2}$ presented in the logarithmic scale), b) Scatter plot derived from the white square region in (a), c) magnified region from the red square in (b). d-f) The same as (a-c), but for $Z=8 \mu \mathrm{m}$. Each cross-point in the scatter maps represents one fluorescent styrene oligomer detected in one exposure frame $(100 \mathrm{~ms})$.

\section{Optimization of the localization parameters with the emitter tracking algorithm}

In our recent work we have described the procedure to determine the survival times of individual fluorescent emitters by using the emitter tracking algorithm. ${ }^{6}$ The algorithm takes into account subsequent localizations in time (blinking gap parameter) and space (pixel jump parameter) and determines whether they originate from the same or different molecule. This is schematically illustrated in Figure S5. If the subsequent localized events (localizations ) appear within $57 \mathrm{~nm}$ of distance and within $2 \mathrm{~s}$ in time, they are counted as one emitter (one fluorescent product molecule). The determined numbers are a good physical approximation of the analyzed data set. This is due to the fact that the many photostable emitters appear highly localized (see Figure S4) and with shorter blinking times (mostly bellow $2 \mathrm{~s}$ ). The purpose of this analysis is to clearly identify the "hotspots" of fluorescence activity that are attributed to the formation of the highly photostable emitters. The complexity of fluorescence blinking and survival time distributions cannot be quantitatively determined by our analysis. Instead, the applied algorithm relies on the statistically sound description of the large number of molecules. It should be noted that the localization parameters in this analysis differ from the ones determined previously for the oligomerization of furfuryl alcohol. ${ }^{6,7}$ This is due to a different nature of fluorescent species and significantly short survival time distributions of individual emitters. The long survival times of the trimeric styrene products and their blinking shifts the distribution towards higher values of the blinking gap parameter. 

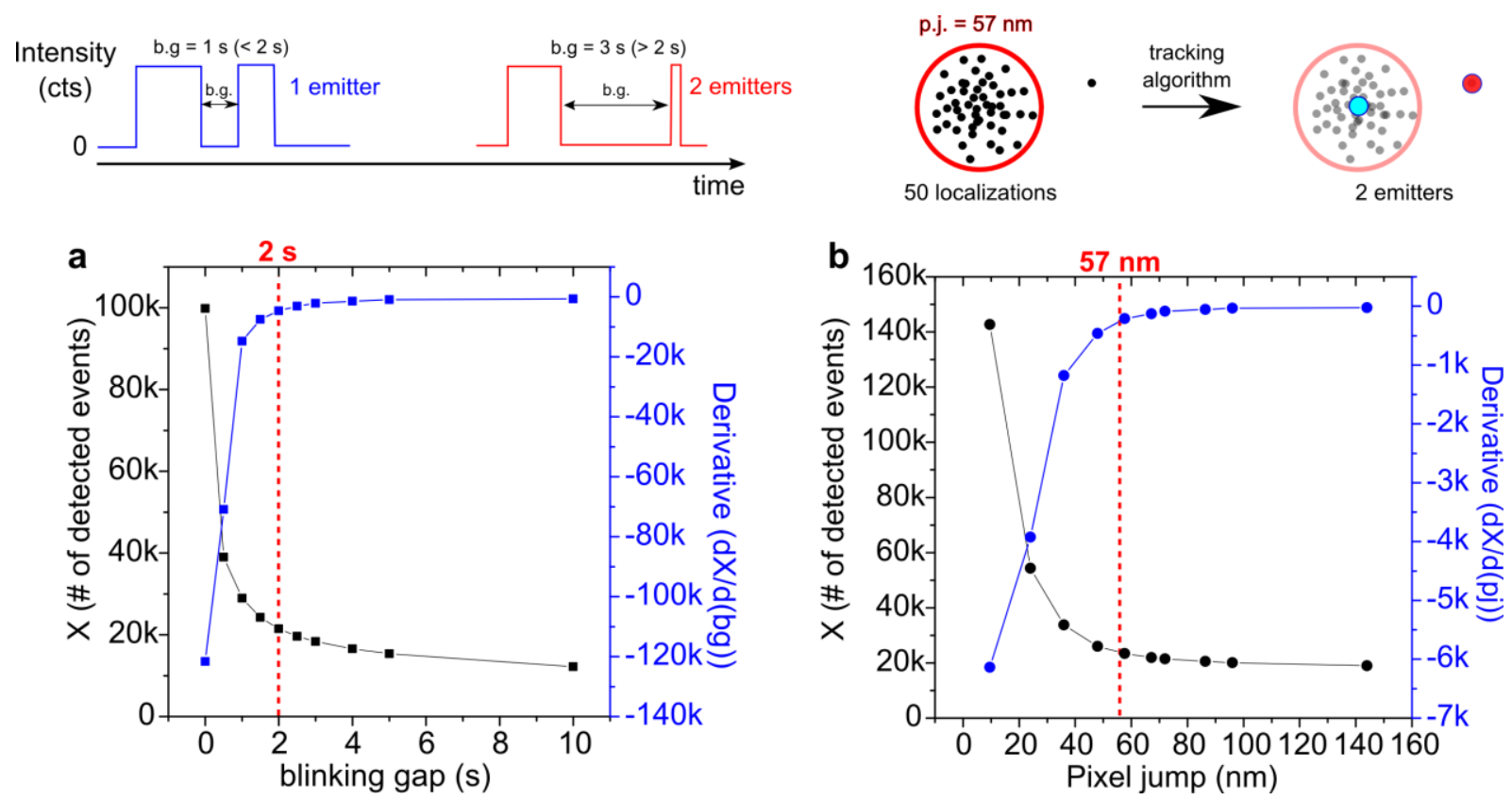

Figure S5. a) Number of detected emitters (in black) and its first derivative (in blue) as a function of the blinking gap parameter. Based on this, the blinking gap was determined to be $2 \mathrm{~s}$; pixel jump value was $72 \mathrm{~nm}$ in all localizations. b) Number of detected emitters (in black) and its first derivative (in blue) as a function of the pixel jump. Based on this, the pixel jump was determined to be $57 \mathrm{~nm}$; The blinking gap value was $2 \mathrm{~s}$ in all localizations.
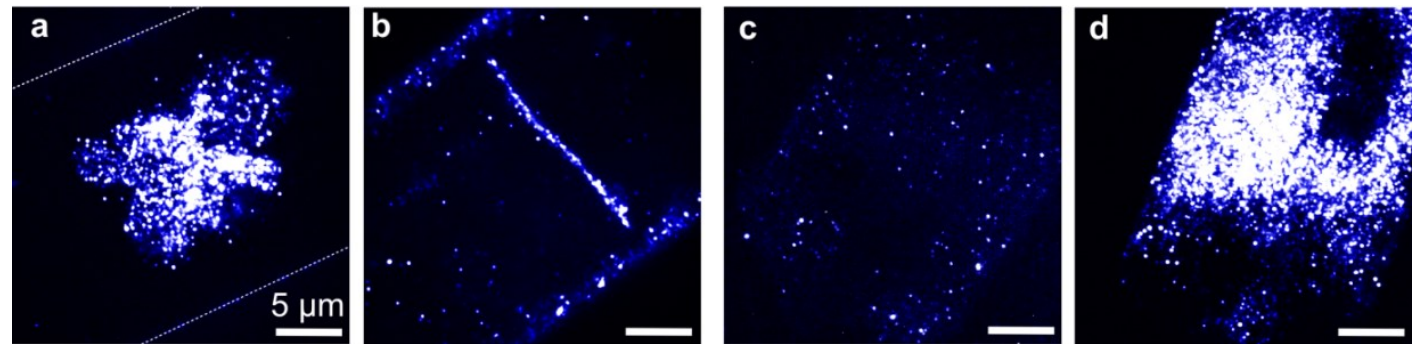

Figure S6. Intraparticle $(\mathrm{a}, \mathrm{b})$ and interparticle heterogeneities $(\mathrm{c}, \mathrm{d})$ determined by SOFI analysis. ${ }^{8,9}$ a,b) the reactivity of parent zeolite H-ZSM-5 crystals with significant intra-crystalline defects, recorded for the oligomerization of 4 - methoxystyrene in heptane $(70 \mathrm{mM})$ at $Z=4 \mu \mathrm{m}$. c,d) The fluorescence response at the surface of two steamed zeolite H-ZSM-5 crystals reacted with 4fluorostyrene in heptane $(80 \mathrm{mM})$. All images represent accumulated fluorescence signal reconstructed with SOFI analysis. Brighter the color, the more photoactive the region of interest. 


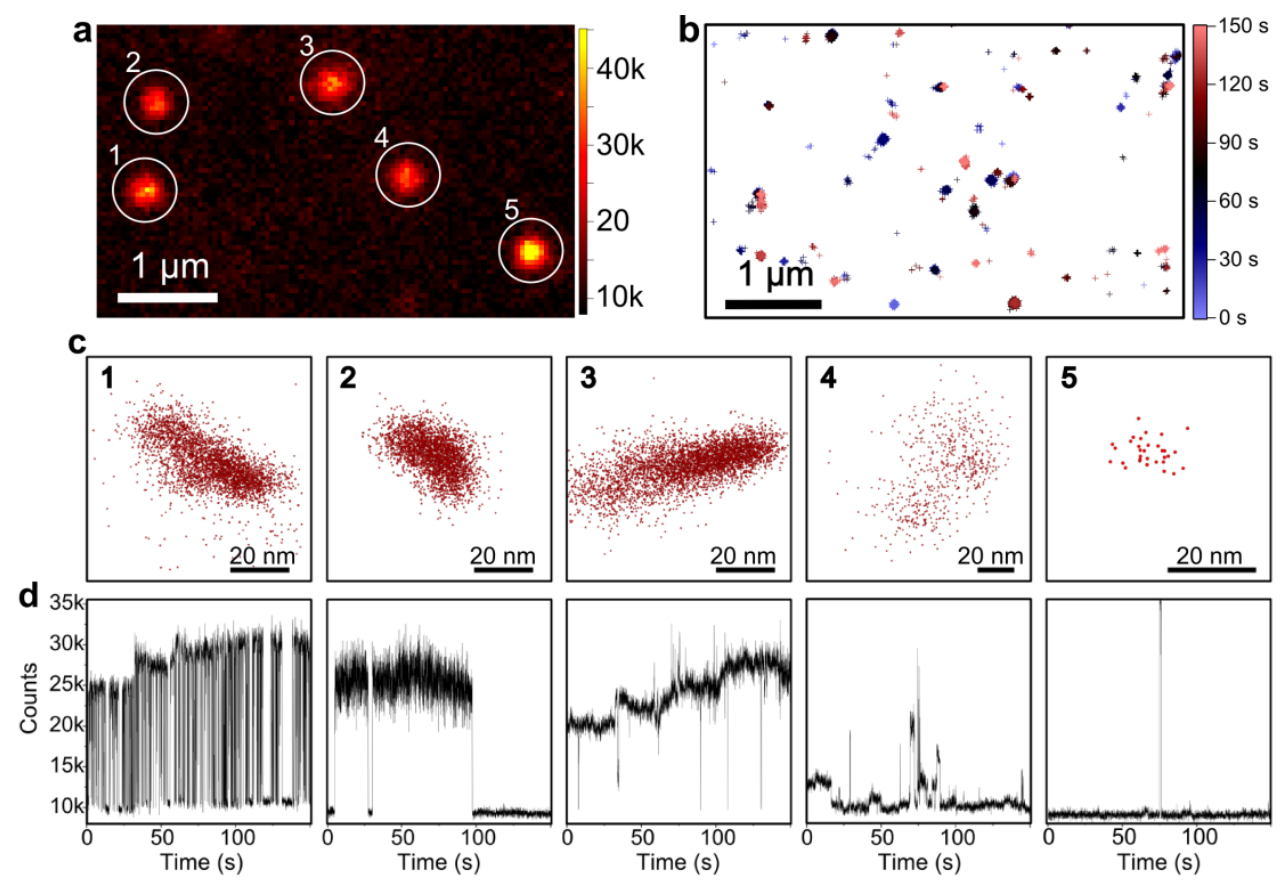

Figure S7. Examples of single molecule fluorescence intensity trajectories recorded during exposure of the steamed zeolite H-ZSM-5 crystal to $30 \mu \mathrm{M}$ solution of 4-methoxystyrene in heptane. a) Single $30 \mathrm{~ms}$ frame indicating 5 fluorescent events presented in (c) and (d). b) Scatter plot of all photo-events accumulated during a time frame of $150 \mathrm{~s}$. Color-coded bar represents the time of detection. c) Scatter plots of the photo-events labelled in (a), and d) corresponding photo-trajectories of the emitters shown in (c).

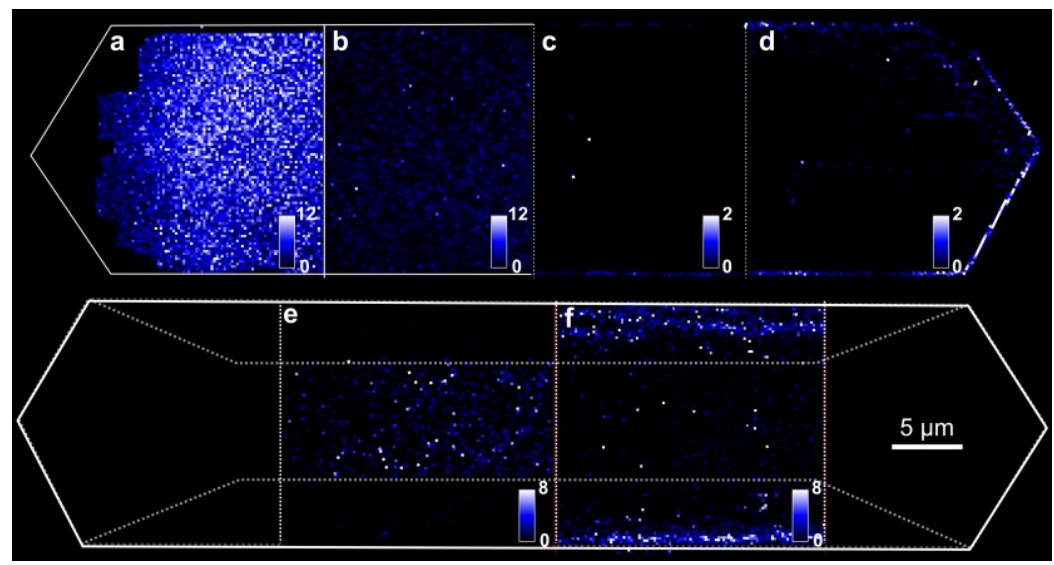

Figure S8. a-d) Comparison of the fluorescence activities of 4-fluorostyrene recorded close to the surface of the parent zeolite H-ZSM- 5 crystals $(Z=0)$ by using a $532 \mathrm{~nm}$ excitation; a) edge after 25 min (solvent-free), b) middle after 15 min (solvent-free), c) middle in 1-butanol (0.085 M) after 30 $\mathrm{min}$, and d) edge in 1-butanol $(0.085 \mathrm{M})$ after $40 \mathrm{~min}$. e, f) Fluorescence activity of zeolite ZSM-5 crystals in solvent-free 4-fluorostyrene recorded at $\mathrm{Z}=4 \mu \mathrm{m}$ for e) parent zeolite H-ZSM-5 crystal, and f) steamed zeolite H-ZSM-5 crystal. The dotted lines indicate the subunit boundaries of the zeolite crystal at focal depth of $Z=4 \mu \mathrm{m}$. The color scale bars indicate the number of detected fluorescent events per $200 \times 200 \mathrm{~nm} 2$ normalized to the recording times of $100 \mathrm{~s}$. 


\section{Supplementary References}

(1) Kox, M. H. F.; Stavitski, E.; Weckhuysen, B. M. Angew. Chem. Int. Ed. 2007, 46, 3652.

(2) Stavitski, E.; Kox, M. H. F.; Weckhuysen, B. M. Chem. Eur. J. 2007, 13, 7057.

(3) Cozens, F. L.; Bogdanova, R.; Régimbald, M.; García, H.; Martí, V.; Scaiano, J. C. J. Phys. Chem. B 1997, 101, 6921 .

(4) Fornés, V.; García, H.; Martí, V.; Fernández, L. Tetrahedron 1998, 54, 3827.

(5) Sprung, C.; Weckhuysen, B. M. J. Am. Chem. Soc. 2015, 137, 1916.

(6) Ristanović, Z.; Hofmann, J. P.; De Cremer, G.; Kubarev, A. V.; Rohnke, M.; Meirer, F.; Hofkens, J.; Roeffaers, M. B. J.; Weckhuysen, B. M. J. Am. Chem. Soc. 2015, 137, 6559.

(7) Liu, K.-L.; Kubarev, A. V.; Van Loon, J.; Uji-i, H.; De Vos, D. E.; Hofkens, J.; Roeffaers, M. B. J. ACS Nano 2014, 8, 12650.

(8) Dedecker, P.; Mo, G. C. H.; Dertinger, T.; Zhang, J. Proc. Natl. Acad. Sci. 2012, 109, 10909.

(9) Ristanović, Z.; Kerssens, M. M.; Kubarev, A. V.; Hendriks, F. C.; Dedecker, P.; Hofkens, J.; Roeffaers, M. B. J.; Weckhuysen, B. M. Angew. Chem. Int. Ed. 2015, 54, 1836. 